\title{
Spectrum Sensing in Dual Polarized Fading Channels for Cognitive SatComs
}

\author{
Shree Krishna Sharma, Symeon Chatzinotas, and Björn Ottersten \\ SnT - securityandtrust.lu, University of Luxembourg \\ Email:\{shree.sharma, symeon.chatzinotas, bjorn.ottersten\}@uni.lu
}

\begin{abstract}
Next generation networks are moving towards the convergence of mobile, fixed and broadcasting services in one standard platform, which requires the co-existence of satellite and terrestrial networks in the same spectrum. This framework has motivated the concept of cognitive Satellite Communication (SatComs). In this aspect, the problem of exploiting Spectrum Sensing (SS) techniques for a dual polarized fading channel is considered. In this paper, the performance of Energy Detection (ED) technique is evaluated in the context of a co-existence scenario of a satellite and a terrestrial link. Diversity combining techniques such as Equal Gain Combining (EGC) and Selection Combining (SC) are considered to enhance the SS efficiency. Furthermore, analytical expressions for probability of detection $\left(P_{d}\right)$ and probability of false alarm $\left(P_{f}\right)$ are presented for these techniques in the considered fading channel and the sensing performance is studied through analytical and simulation results. Moreover, the effect of Cross Polar Discrimination (XPD) on the sensing performance is presented and it is shown that SS efficiency improves for low XPD.
\end{abstract}

Index Terms-Sensing efficiency, Energy detection, Dual polarized channel, Cross polar discrimination

\section{INTRODUCTION}

Recently, the possibility of co-existence of future satellite networks with terrestrial systems leading towards the convergence of mobile, fixed and broadcasting systems has been an important research topic [1] [2]. Different fundamental physical constraints such as power requirements, terrain and infrastructure obstructions, antenna types etc. may cause the terrestrial communication to be dependent on satellite communication. The satellite technology is able to overcome the drawbacks experienced by terrestrial systems to extend the coverage. Therefore, the hybrid/integrated systems can be considered as a means to provide true integration [2]. Satellite communication plays a significant role in supporting hybrid/integrated architectures for future wireless networks. There has been continued pressure on satellite bands, especially in $\mathrm{L}$ and $\mathrm{C}$ bands due to the introduction of new terrestrial services such as $3 \mathrm{G}$ mobile telephony, WiMax and WiFi services [3]. Due to the limited and expensive bandwidth, Cognitive Radio (CR) can be an efficient way to increase spectrum efficiency for future satellite networks. In this direction, satellite and terrestrial networks can be considered as primary and secondary systems respectively or vice versa.

The most common cognitive techniques in literature can be categorized into interweave or SS, underlay, and overlay techniques [4]. In SS only techniques, Secondary Users (SUs) are allowed to transmit whenever Primary Users (PUs) do not use that specific band, whereas in underlay techniques, SUs are allowed to transmit as long as they meet the interference constraint of PUs. SS is one important mechanism for the spectrum awareness required by CRs. Among many available SS techniques in the literature, Energy Detection (ED) is the most common way of SS because of its low computational and implementation complexities [5-7]. The concepts of spectrum sharing in the frequency, time and spatial domains have been considered in much literature but the polarization domain has not been fully exploited. In the existing literature, the polarization domain has been used for diversity and multiplexing purposes [8-10].

The polarization domain can be treated as an additional degree of freedom to explore efficient SS and transmission schemes in the context of cognitive SatComs. Dual polarization plays a significant role in satellite systems to increase the efficiency of the available bandwidth since two independent signals can be transmitted on the same spectrum by means of two orthogonal polarizations, hence doubling the available spectrum. Furthermore, two orthogonal polarizations can be treated as two different data streams (colors) and dual polarization can be used as an additional color in the frequency planning process of multi-beam satellites and terrestrial cellular networks [11]. From the cognitive point of view, if primary system is designed to transmit in one polarization, secondary system can be designed to transmit in another polarization using the polarization domain as an interference mitigation tool towards primary systems.

Dual polarized channel has been considered in satellite links and terrestrial links to increase the spectrum efficiency as well as to remove the practical difficulty of spatial separation required by antennas in Multiple Input Multiple Output (MIMO) links [8] [11]. The polarization domain has been exploited for SS purpose in a dual polarized Additive White Gaussian Noise (AWGN) channel in [12]. The use of dual polarized antennas has become a promising cost-effective and space-effective alternative for enhancing the reliability of transmission by creating polarization diversity [13]. However, wireless fading channels may reduce the orthogonality of dual polarized channels causing cross polarization effects.

The performance of ED based SS has been carried in Rayleigh, Rician and Nakagami fading channel in much literature [5-7]. According to authors' knowledge, dual polarized fading channel has not been considered in literature for SS purpose. Since dual polarized channel is getting more impor- 
tance in current state of art related to cognitive communication [11] [14], it remains an open challenge to explore the best SS technique in this channel. Furthermore, cross polarization effects impose a challenge to carry out SS in a dual polarized fading channel. This is the main motivation for this paper. The main contribution of this paper is performance analysis of a ED based SS technique in a dual polarized fading channel using different combining techniques. In this paper, we start with single antenna case and we review analytical expressions for $P_{d}$ and $P_{f}$ for AWGN and Rayleigh fading channels. Then we deal with uni-polarized MIMO channel which acts as a building block for the dual polarized channel and we include the cross correlation in channel to include the effect of XPD in a dual polarized fading channel.

The remainder of this paper is organized as follows: section II presents the practical application scenario and provides the system and signal model. Section III provides the theoretical basis of traditional ED technique in the context of single antenna in a wireless fading channel. Section IV describes the ED problem in uni-polarized MIMO and dual polarized scenarios. Combining techniques SC and EGC for SS are discussed in section $\mathrm{V}$ along with the theoretical expressions. Section VI describes the simulation environment and presents theoretical as well as simulation results. Section VII concludes this paper.

\section{System And Signal Model}

\section{A. Use case}

The hybrid satellite-terrestrial system as shown in Fig. 1 can be considered as one practical scenario. In this scenario, terrestrial WiMax network can be considered as a primary system and satellite network operating in C-band as a secondary sytem. The new terrestrial wireless service WiMax defined by the ITU uses the same C-band spectrum and in many cases, extended $\mathrm{C}$ band $(3.4-3.7) \mathrm{GHz}$ band has been allotted to WiMax service and some regulators are looking to deploy WiMax in standard C-band $(3.7-4.2) \mathrm{GHz}$ too [15]. In terrestrial WiMax system, the microwave backhaul link between base stations can be used as a primary link and satellite uplink from a satellite terminal to the satellite can be considered as a secondary link. This scenario can be beneficial for satellite operators to take advantage of terrestrial licensed spectrum in secondary basis. The use of dual polarization in satellite transmission as well as in terrestrial transmission has been exploited for creating polarization diversity [11] [16] [17], hence, dual polarized antennas can be considered on both satellite and terrestrial networks for this cognitive scenario. The interference from terrestrial WiMax BS antenna to the satellite can be considered to be negligible due to large distance, whereas interference from satellite terminal to terrestrial receiver is strong and needs to be taken into account to meet the interference constraint of primary terrestrial users. More analysis about interference modeling between satellite terminals and terrestrial BS antenna has been presented in [18].

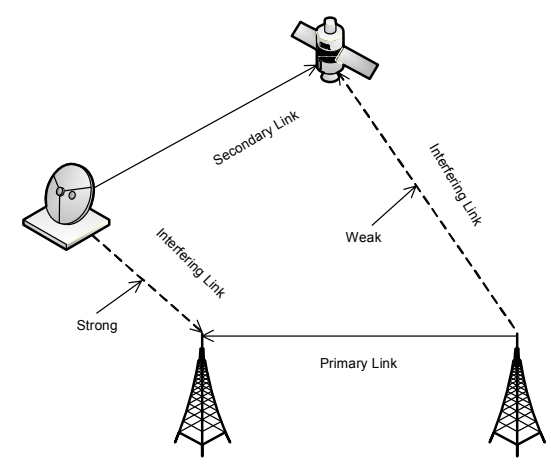

Fig. 1: Hybrid satellite terrestrial cognitive scenario

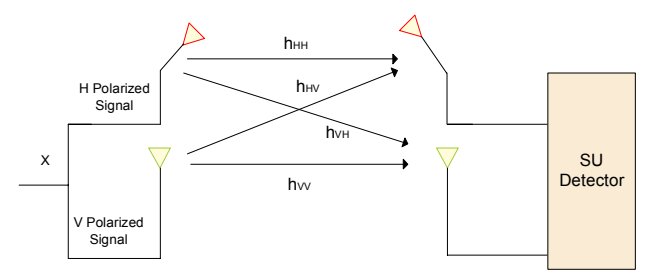

Fig. 2: System Model

\section{B. Model Description}

We consider a cognitive scenario with a SU receiver and a PU transmitter both equipped with dual polarized antennas. In this analysis, we consider interweave cognitive technique rather than underlay and secondary transmission is allowed using PU channel only when PU is not active. Furthermore, it can be considered that the same symbol can be aligned to two orthogonal polarization states and be transmitted through different dimensions from a PU transmitter. The system model is illustrated in Fig. 2. The received signal at SU receiver can be written in the following form:

$$
\mathbf{y}=\mathbf{H}_{d} \mathbf{x}+\mathbf{w}
$$

where $\mathbf{H}_{d}$ is $(2 \times 2)$ channel matrix, $\mathbf{x}$ is $(2 \times 1)$ dual polarized signal and $\mathbf{w}$ is Additive White Gaussian Noise (AWGN) vector including independent noise coefficients at two branches of dual polarized antenna. The channel matrix $\mathbf{H}_{d}$ can be written as:

$$
\mathbf{H}_{d}=\left[\begin{array}{cc}
h_{H H} & h_{H V} \\
h_{V H} & h_{V V}
\end{array}\right]
$$

where $h_{m n}$ denotes the channel coefficient on channel $m n$. Let $H_{0}$ denote the hypothesis of PU being absent and $H_{1}$ denotes hypothesis of active PU. The PU signal detection problem can be formulated in terms of following binary hypothesis testing problem.

$$
\begin{aligned}
& H_{0}: \mathbf{y}(n)=\mathbf{w}(n) \quad \text { PU absent } \\
& H_{1}: \mathbf{y}(n)=\mathbf{H}_{d} \mathbf{x}(n)+\mathbf{w}(n) \quad \mathrm{PU} \text { present }
\end{aligned}
$$

where $n=1,2, \ldots, u, u$ being the product of observation duration $T$ and bandwidth $B$ i.e. $u=T B$. We consider Rayleigh fading channel in this work as a first step towards analyzing the SS performance in different fading channels since there is no line of sight path between satellite terminal and terrestrial 
transmitter/receiver. We consider SC and EGC techniques in energy level instead of co-phasing and adding the signals from different antennas as in conventional diversity schemes. These definitions are similar to the square law selection (SLS) and square law combining (SLC) terms used in [6]. Similar analysis has been carried out in [19]. Furthermore, it can be noted that conventional diversity combining techniques such as Maximum Ratio Combining (MRC) and EGC techniques seem to be difficult to apply for unknown PU signal detection due to weak PU signal as well as no co-operation from source in the context of cognitive Satcoms.

\section{ENERGY DETECTION FOR SINGLE ANTENNA CASE}

The ED problem was first studied in [20] for deterministic signals transmitted over a flat band-limited Gaussian noise channel. The performance of SS methods degrades due to fading effects in a wireless channel. The ED problem for signals transmitted over a variety of fading channels using different receive diversity schemes has been considered in [5], [6], [7] [19]. In practical scenarios, the total fading involves the combined effect of two independent processes: small-scale fading and large scale fading i.e. multi-path and shadowing effects [21]. In case of a satellite channel, lognormal fading due to shadowing, rain fading and other atmospheric effects should be taken into consideration [22]. Since we are considering our system model with respect to satellite-terrestrial hybrid scenario, we focus on the terrestrial wireless channel as well for sensing terrestrial channel with a satellite cognitive terminal.

Before considering fading scenario, let's start with the AWGN case. The decision statistic $(Y)$ of the energy detector follows a central chi-square $\left(\chi^{2}\right)$ distribution with $2 u$ degrees of freedom under hypothesis $H_{0}$. Under hypothesis $H_{1}$, signal and noise both are present and decision statistic $Y$ follows a non-central $\chi^{2}$ distribution with $2 u$ degrees of freedom (dof) and non-centrality parameter $2 \gamma, \gamma$ being signal to noise ratio (SNR) of the PU signal i.e.

$$
Y \sim \begin{cases}\chi_{2 u}^{2}, & H_{0} \\ \chi_{2 u}^{2}(2 \gamma), & H_{1}\end{cases}
$$

The PDF of $Y$ under $H_{0}$ and $H_{1}$ can be written as [6]:

$$
Y= \begin{cases}\frac{1}{2^{u} \Gamma(u)} y^{u-1} e^{-\frac{y}{2}}, & H_{0} \\ \frac{1}{2}\left(\frac{y}{2 \gamma}\right)^{\frac{u-1}{2}} e^{-\frac{2 \gamma+y}{2}} I_{u-1}(\sqrt{2 \gamma y}), & H_{1}\end{cases}
$$

where $\Gamma($.$) is the gamma function and I_{n}($.$) is the n$-th order modified Bessel function of first kind. Then $P_{f}$ and $P_{d}$ can be computed by [23];

$$
\begin{aligned}
P_{f} & =\operatorname{Pr}\left(Y>\lambda \mid H_{0}\right), \\
P_{d} & =\operatorname{Pr}\left(Y>\lambda \mid H_{1}\right),
\end{aligned}
$$

where $\lambda$ is the decision threshold. The expression for $P_{f}$ can be computed using (5) to evaluate (6) and can be written as:

$$
P_{f}=\frac{\Gamma\left(u, \frac{\lambda}{2}\right)}{\Gamma(u)}
$$

where $\Gamma(.,$.$) is the incomplete gamma function. Similarly, the$ expression for $P_{d}$ can be computed using (5) to evaluate (7) and can be written as:

$$
P_{d}=Q_{u}(\sqrt{2 \gamma}, \sqrt{\lambda})
$$

where $Q_{u}(.,$.$) is the generalized Marcum Q-function. The$ expressions in (8) and (9) do not include the effect of channel fading. For the fading scenario, the expression for $P_{f}$ becomes same as (8) since it is independent of SNR, hence, independent of any fading. $P_{d}$ in any fading channel can be calculated by averaging the conditional $P_{d}$ as given by (9) over the fading distribution [5]. For the case of small-scale fading, the distribution of the envelope of the received signal is generally considered to be Rayleigh distributed, which makes the received power distribution to be exponential [19]. The instantaneous SNR $\gamma$ of a signal having Rayleigh distributed amplitude follows an exponential PDF given by [5];

$$
f(\gamma)=\frac{1}{\bar{\gamma}} \exp \left(-\frac{\gamma}{\bar{\gamma}}\right) \quad \gamma \geq 0
$$

where $\bar{\gamma}$ is the average SNR. The average $P_{d}$ in Rayleigh fading case, denoted as $\bar{P}_{d r}$, can be calculated by averaging (9) over (10), which can be written as:

$$
\bar{P}_{d r}=\int_{0}^{\infty} Q_{u}(\sqrt{2 \gamma}, \sqrt{\lambda}) f(\gamma) d \gamma
$$

Letting $x=\sqrt{2 \gamma}$, the above expression can be written as:

$$
\bar{P}_{d r}=\frac{1}{\bar{\gamma}} \int_{0}^{\infty} Q_{u}(x, \sqrt{\lambda}) x e^{-\frac{x^{2}}{2 \bar{\gamma}}} d x
$$

By solving above integral problem using (12) from [24], the closed form solution for $\bar{P}_{d r}$ can be written as:

$$
\begin{aligned}
\bar{P}_{d r}=e^{\frac{-\lambda}{2}} & \sum_{n=0}^{u-2} \frac{1}{n !}\left(\frac{\lambda}{2}\right)^{n}+\left(\frac{1+\bar{\gamma}}{\bar{\gamma}}\right)^{u-1} \\
\times & {\left[e^{-\frac{\lambda}{2(1+\bar{\gamma})}}-e^{-\frac{\lambda}{2}} \sum_{n=0}^{u-2} \frac{1}{n !}\left(\frac{\lambda \bar{\gamma}}{2(1+\bar{\gamma})}\right)^{n}\right] }
\end{aligned}
$$

\section{ENERGY DETECTION FOR DUAL ANTENNA CASE}

\section{A. Uni-polarized MIMO channel}

In this channel, all antennas are identically polarized and this channel can be considered as a bulding block for dual polarized channels. The MIMO channel matrix $\mathbf{H}$ can be defined as:

$$
\mathbf{H}=\left[\begin{array}{ll}
h_{11} & h_{12} \\
h_{21} & h_{22}
\end{array}\right]
$$

The channel coefficients $h_{11}, h_{12}, h_{21}$, and $h_{22}$ are considered to be c.c.s. i.i.d. random variables modeling Rayleigh fading. From the polarization point of view, the matrix $\mathbf{H}$ denotes uni-polarized channel matrix i.e. all antennas are identically polarized. Then the binary hypothesis testing problem can be written as:

$$
\begin{aligned}
& H_{0}: \mathbf{y}(n)=\mathbf{w}(n) \quad \mathrm{PU} \text { absent } \\
& H_{1}: \mathbf{y}(n)=\mathbf{H x}(n)+\mathbf{w}(n) \quad \mathrm{PU} \text { present }
\end{aligned}
$$


where $\mathbf{x}(n)=\left[\begin{array}{c}X(n) \\ X(n)\end{array}\right], X(n)$ being $n$-th transmitted symbol. In this scenario, fading signal amplitude is found to be Rayleigh distributed with parameter $\sigma=2$ instead of Rayleigh distribution with parameter $\sigma=1$ in single antenna case. We consider this factor to derive the theoretical expression for $P_{d}$ in case of a MIMO channel in the next section.

\section{B. Dual polarized channel}

Two branches of a dual polarized antenna can be considered to receive $\mathrm{H}$-polarized and $\mathrm{V}$-polarized signals coming from PU transmit antenna. In this work, we consider the enhancement of diversity rather than multiplexing using diversity combining techniques. For this purpose, the same symbol can be aligned to horizontal and vertical orthogonal polarization for transmission purpose in PUs' transmitter. Dual polarized channel can be regarded as a $2 \times 2$ MIMO spatial channel if we exclude the polarization effects. The exact modeling of a dual polarized channel is quite complex and it depends on the following factors [14].

- Spatial separation between two antennas at both transmit and receive sides.

- Cross-polar isolation (XPI) of transmit and receive antennas: Its value is taken as infinite for perfect antennas.

- Spatial separation in channel: This is taken into account as in uni-polarized MIMO channel.

- Cross-polar ratio (XPR) of the channel: This becomes zero for perfect orthogonality of two polarized channels.

- Cross Polarization discrimination (XPD): It is the combined effect of XPI and XPR.

In the context of analysis in this paper, we consider the following scenario. Let's assume that co-located orthogonally polarized antennas are placed on both transmit and receive sides and XPI factor of both antennas is infinite. By making these assumptions, we are removing the effects of spatial correlation due to antenna separation and cross-polar reception due to imperfection of antennas on both sides. The channel matrix in this condition can be modeled in the following way:

$$
\mathbf{H}_{d}=\left[\begin{array}{ll}
h_{H H} & h_{H V} \\
h_{V H} & h_{V V}
\end{array}\right]=\mathbf{X} \odot \mathbf{H}
$$

where $\mathbf{H}$ is uni-polarized channel matrix as considered in the above section, $\odot$ denotes Hadamard product, $E\left[\left|h_{H H}\right|^{2}\right]=$ $E\left[\left|h_{V V}\right|^{2}\right]=1, E\left[\left|h_{V H}\right|^{2}\right]=E\left[\left|h_{H V}\right|^{2}\right]=\sqrt{\chi_{c x}}, \chi_{c x}$ being XPR and

$$
\mathbf{X}=\left[\begin{array}{cc}
1 & \sqrt{\chi_{c x}} \\
\sqrt{\chi_{c x}} & 1
\end{array}\right]
$$

The variable $\chi_{c x}$ quantifies the inverse of XPD in case of infinite XPI and varies in range $[0,1]$, since $0 \leq X P D<\infty$ [10]. It can be expressed as: $\chi_{c x}=\frac{\chi_{c x, H}+\chi_{c x, V}}{2}$ with $\chi_{c x, V}=$ $\frac{E\left|h_{H V}\right|^{2}}{E\left|h_{V V}\right|^{2}}$ and $\chi_{c x, H}=\frac{E\left|h_{V H}\right|^{2}}{E\left|h_{H H}\right|^{2}}$. The decision statistic to check the hypothesis problem given in (3) for a dual polarized channel depends on the type of combining technique used in combining two branch signals.

\section{COMBINING TECHNIQUES FOR DUAL POLARIZED CHANNEL}

\section{A. Selection Combining}

The block diagram of SC technique for SS using dual polarized antenna has been shown in Fig. 3. Two branch signals are passed through the filter to limit the noise power and then passed through squared and integrator devices. The energies of received signals in each branch are computed by taking samples within a certain period $T$ and the detector selects the highest energy between them. The decision statistic for SC can be expressed as: $Y_{s c}=\max \left(Y_{H}, Y_{V}\right)$, where $Y_{H}$ and $Y_{V}$ are decision statistics calculated for H-polarized branch and V-polarized branch respectively. Under $H_{0}$, the expression for $P_{f}$ for SC, $P_{f, s c}$, becomes different than that for single antenna case and can be evaluated using CDF of $Y_{s c}$ given $H_{0}$ i.e. $F_{Y_{s c}}$, can be written as [6]:

$$
P_{f, s c}=1-F_{Y_{s c}}\left(\lambda \mid H_{0}\right)=1-\left[1-\frac{\Gamma\left(u, \frac{\lambda}{2}\right)}{\Gamma(u)}\right]^{2}
$$

Similarly, $P_{d}$ for SC i.e. $P_{d, s c}$ in AWGN channel under $H_{1}$ can be written as:

$P_{d, s c}=1-\left[1-Q_{u}\left(\sqrt{2 \gamma_{H}}, \sqrt{\lambda}\right)\right]\left[1-Q_{u}\left(\sqrt{2 \gamma_{V}}, \sqrt{\lambda}\right)\right]$

The average value of $\bar{P}_{d, s c}$ over 2 independent Rayleigh branches can be evaluated in the following way [6]:

$$
\begin{aligned}
\bar{P}_{d, s c}= & 1-\int_{0}^{\infty}\left[1-Q_{u}\left(\sqrt{2 \gamma_{H}}, \sqrt{\lambda}\right)\right] f\left(\gamma_{H}\right) d\left(\gamma_{H}\right) \\
& \times \int_{0}^{\infty}\left[1-Q_{u}\left(\sqrt{2 \gamma_{V}}, \sqrt{\lambda}\right)\right] f\left(\gamma_{V}\right) d\left(\gamma_{V}\right)
\end{aligned}
$$

The above equation when combined with $\bar{P}_{d r}$ expression from (13) can be written as:

$$
\bar{P}_{d, s c}=1-\left(1-\bar{P}_{d r}\left(\bar{\gamma}_{H}, 2 u\right)\right) \times\left(1-\bar{P}_{d r}\left(\bar{\gamma}_{V}, 2 u\right)\right)
$$

In the considered dual polarized scenario, the expression for $P_{f}$ remains same as (18) because the noise processes are same as in single input multiple output (SIMO) scenario. The distribution of amplitude of both $\mathrm{H}$-polarized and V-polarized signals is Rayleigh with varying parameter $\sigma=1+\sqrt{\chi_{c x}}$ instead of Rayleigh distribution with parameter $\sigma=1$ in SIMO scenario. Since the value of $\chi_{c x}$ ranges from 0 to 1 , the value of $\sigma$ ranges from 1 for SIMO case to 2 for ideal MIMO case. This effect can be reflected in $P_{d}$ expression by scaling $\bar{\gamma}_{H}$ and $\bar{\gamma}_{V}$ by the factor $\sigma$ in (21) and can be written as:

$$
\bar{P}_{d, s c}=1-\left(1-\bar{P}_{d r}\left(\sigma * \bar{\gamma}_{H}, 2 u\right)\right) \times\left(1-\bar{P}_{d r}\left(\sigma * \bar{\gamma}_{V}, 2 u\right)\right)
$$

\section{B. Equal Gain Combining (EGC)}

The block diagram of EGC technique has been shown in Fig. 3. This technique combines the energy of two branches with equal gain i.e. it simply adds energies of two branches of a dual polarized antenna. The final decision statistic using this technique can be written as: $Y_{e g c}=Y_{H}+Y_{V}$. Under 


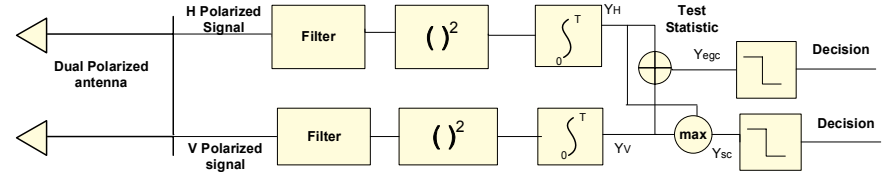

Fig. 3: Block diagram of SC and EGC techniques for SS

$H_{0}$ hypothesis in AWGN channel, adding 2 i.i.d. central chisquare variates, each with $2 u$ dof and unit variance results in another central chi-square variate with $4 u$ dof with the same variance [6]. Therefore, $P_{f}$ for EGC, $P_{f, e g c}$ can be written as:

$$
P_{f, e g c}=\frac{\Gamma\left(2 u, \frac{\lambda}{2}\right)}{\Gamma(2 u)}
$$

The decision statistic $Y_{e g c}$ under hypothesis $H_{1}$ in AWGN channel follows a non-central chi-square $\chi^{2}$ distribution with $4 u$ degrees of freedom and non-centrality parameter $2 \gamma_{e g c}$. The expression for $P_{d}$ in AWGN channel for EGC, $P_{d, e g c}$, can be written as:

$$
P_{d, e g c}=Q_{2 u}\left(\sqrt{2 \gamma_{e g c}}, \sqrt{\lambda}\right)
$$

where $\gamma_{e g c}$ is the SNR of the combined signal. For Rayleigh fading scenario, considering 2 i.i.d. Rayleigh fading branches of a dual polarized antenna, the PDF of $\gamma_{e g c}$ can be written as [5]:

$$
f\left(\gamma_{e g c}\right)=\frac{1}{\bar{\gamma}_{e g c}^{2}} \gamma_{e g c} \exp \left(\frac{-\gamma_{e g c}}{\bar{\gamma}_{e g c}}\right)
$$

The average value of $\bar{P}_{d}, e g c$ in Rayleigh fading channel can then be obtained by averaging (24) over (25) as:

$$
\bar{P}_{d, e g c}=\int_{0}^{\infty} Q_{2 u}\left(\sqrt{2 \gamma_{e g c}}, \sqrt{\lambda}\right) f\left(\gamma_{e g c}\right) d\left(\gamma_{e g c}\right)
$$

The closed form for above equation can be written in the following form. It should be noted that we present $\bar{P}_{d, \text { egc }}$ for 2 branch scenario of a dual polarized antenna instead of Lbranch scenario given in [6].

$\bar{P}_{d, e g c}=G+K^{2} e^{\frac{-\lambda}{2}}\left[\sum_{i=1}^{2 u-1} \frac{\left(\frac{\lambda}{2}\right)^{i}}{i !}{ }_{1} F_{1}\left(2 ; i+1 ; \frac{\lambda(1-K)}{2}\right)\right]$

where ${ }_{1} F_{1}$ is the confluent hypergeometric function, $K=$ $\frac{1}{1+\bar{\gamma}_{e g c}}$ and

$$
G=e^{\frac{-\lambda K}{2}}\left[1+\frac{K \lambda(1-K)}{2}\right]
$$

In case of dual polarized scenario, the expression for $P_{f}$ remains same as (23). The expression for $P_{d}$ then becomes (27) with modified value of $K$ which can be written as: $K^{\prime}=\frac{1}{1+\sigma \bar{\gamma}_{e g c}}$, where $\sigma=1+\sqrt{\chi_{c x}}$ as defined in earlier subsection. Therefore, the value of of $\bar{P}_{d, e g c}$ in a dual polarized channel depends on the value of XPR and ranges from upper bound for an ideal MIMO channel to lower bound for a SIMO channel.

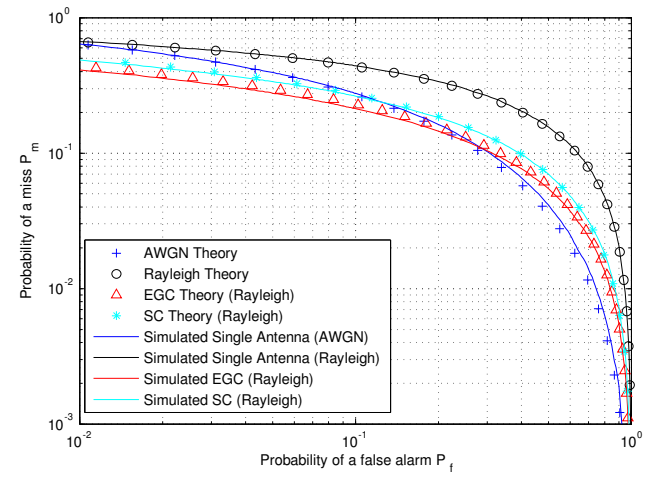

Fig. 4: $P_{m}$ versus $P_{f}$ for different cases $(\mathrm{SNR}=-4 \mathrm{~dB}$, dof $=60$, Number of iterations $(\mathrm{N})=10000$ )

\section{Vi. Simulation Results}

To analyze the sensing performance of considered system, complementary receiver operating characteristics (CROC) curves (probability of a miss, $P_{m}$ versus $P_{f}$ ) are plotted based on both theoretical and simulation results. We consider the following cases for carrying out analysis. 1) First case considers single antenna reception in AWGN channel for reference purpose. 2) Second case considers the single antenna reception with a flat fading Rayleigh channel. 3) In this case, we consider receive diversity with two receiving antenna with SIMO Rayleigh channel. SC and EGC techniques have been considered to combine the signals coming from two branches. 4) This case considers 2 transmit and 2 receive antenna with a $2 \times 2$ Rayleigh fading channel to resemble the channel structure of dual polarized wireless channel. 5) In this case, we include power imbalance in the MIMO channel to reflect XPD effect in a dual polarized channel. Figure 4 shows the theoretical and analytical comparison of CROC curves for cases 1, 2 and 3. The theoretical and simulation results of SC and EGC techniques in a Rayleigh fading channel has been plotted in this figure. From the simulation and analytical results, it can be noted that EGC performs only slightly better than $\mathrm{SC}$ technique in a Rayleigh fading channel. Both techniques perform better than single antenna reception. These results are consistent with the results obtained in literature [5] and we are considering low value of SNR to reflect the weak nature of signals in cognitive Satcoms. Figure 5 shows the comparison of SC and EGC techniques in MIMO and SIMO Rayleigh fading channels for cases 2, 3 and 4. Theoretical and simulation results for EGC and SC techniques have been plotted. From the results, it can be noted that both techniques perform better in a MIMO Rayleigh fading channel than in SIMO scenario.

Figure 6 shows $P_{m}$ versus XPR value for SC and EGC techniques for $P_{f}$ value of 0.07 at two different SNR values of $0 \mathrm{~dB}$ and $-5 \mathrm{~dB}$. From the simulation results, it can be noted that for both techniques $P_{m}$ decreases with the value of $\chi_{c x}$ and these two techniques provide closer detection performance at higher values of SNR. The value of $P_{m}$ becomes minimum for unity value of $\chi_{c x}$ and it becomes maximum when $\chi_{c x}$ equals zero. From these results, it can be inferred that the 


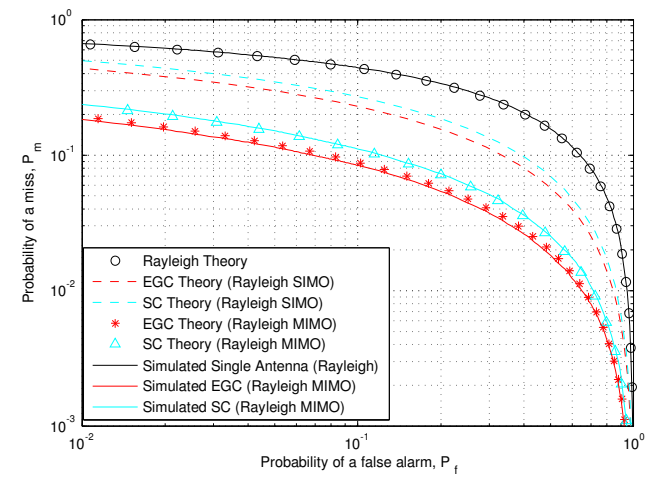

Fig. 5: $P_{m}$ versus $P_{f}$ for SC and EGC techniques in MIMO Rayleigh fading channel $(\mathrm{SNR}=-4 \mathrm{~dB}$, dof $=60, \mathrm{~N}=10000)$

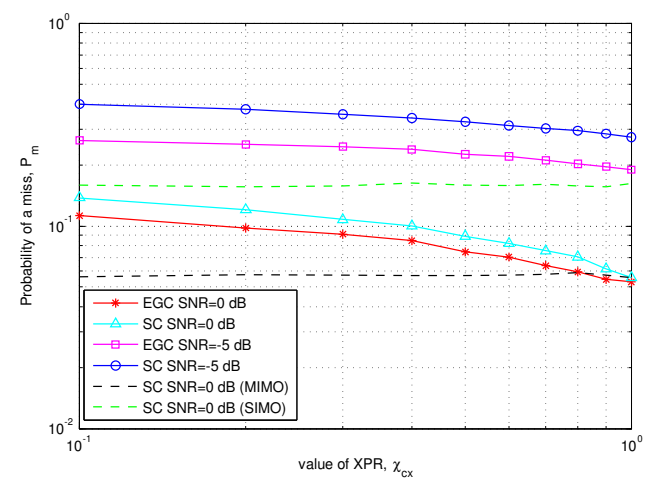

Fig. 6: $P_{m}$ versus XPR for $P_{f}=0.07(\mathrm{dof}=60, \mathrm{~N}=20000)$

sensing performance in a dual polarized channel increases with the value of $\chi_{c x}$ and it ranges from minimum for SIMO channel to maximum for an ideal MIMO channel. Furthermore, in terms of XPD, the detection performance decreases with the value of XPD since $\chi_{c x}$ quantifies the inverse of XPD.

\section{CONCLUSION}

ED based SS technique has been analyzed in a dual polarized fading channel in the context of cognitive Satcoms. EGC and SC techniques have been considered to enhance SS efficiency by combining the received two branch signals at the energy level. The theoretical expressions for $P_{d}$ and $P_{f}$ have been provided for these techniques in AWGN, SIMO and MIMO Rayleigh fading channels. The simulation as well as theoretical results show that the performance of ED technique in a MIMO fading channel is much better than in SIMO and SISO fading channels. Furthermore, the effect of XPR on SS efficiency has been analyzed and it can be concluded that the detection performance in a dual polarized fading channel increases with the value of XPR and its lower and upper bounds occur for a SIMO fading channel and an ideal MIMO fading channel resepctively.

\section{ACKNOWLEDGEMENT}

This work was supported by the National Research Fund, Luxembourg under AFR (Aids Training-Research) grant for $\mathrm{PhD}$ project on "Spectrum Sensing, Resource Allocation and
Resource Management Strategies for Satellite Cognitive Communications", under the CORE project "CO2SAT: Cooperative and Cognitive Architectures for Satellite Networks" and by COST Action IC0902.

\section{REFERENCES}

[1] S. Kota, "Hybrid/integrated networking for NGN services," in 2nd Int. Conf. Wireless VITAE, March 2011, pp. 1 -6.

[2] B. Evans and et al, "Integration of satellite and terrestrial systems in future multimedia communications," IEEE Wireless Commun., vol. 12, no. 5 , pp. $72-80$, oct. 2005 .

[3] S. Kota, G. Giambene, and S. Kim, "Satellite component of NGN: Integrated and hybrid networks," Int. J. Satell. Commun. Network, 2010.

[4] A. Goldsmith, S. Jafar, I. Maric, and S. Srinivasa, "Breaking spectrum gridlock with cognitive radios: An information theoretic perspective," Proc. of the IEEE, vol. 97, no. 5, pp. 894 -914, may 2009.

[5] F. Digham, M.-S. Alouini, and M. Simon, "On the energy detection of unknown signals over fading channels," in IEEE Int. Conf. Commun., vol. 5, may 2003, pp. 3575 - 3579 .

[6] F. F. Digham, M.-S. Alouini, and M. K. Simon, "On the energy detection of unknown signals over fading channels," IEEE Trans. Commun., vol. 55, no. 1 , pp. $21-24$, jan. 2007.

[7] S. Herath, N. Rajatheva, and C. Tellambura, "Energy detection of unknown signals in fading and diversity reception," IEEE Trans. Commun., vol. 59, no. 9, pp. $2443-2453$, september 2011.

[8] B. S. Collins, "Polarization-diversity antennas for compact base stations," Microwave J., vol. 43, no. 1, pp. 76-88, Jan. 2000.

[9] J. Dietrich, C.B., K. Dietze, J. Nealy, and W. Stutzman, "Spatial, polarization, and pattern diversity for wireless handheld terminals," IEEE Trans. Antennas and Propag., vol. 49, no. 9, pp. 1271 -1281, sep 2001.

[10] S. Chatzinotas, D. Christopoulos, and B. Ottersten, "Coordinated multipoint decoding with dual-polarized antennas," in Proc. 7th IWCMC, july 2011, pp. $157-161$.

[11] N. Zorba, M. Realp, M. Lagunas, and A. Perez-Neira, "Dual polarization for MIMO processing in multibeam satellite systems," in Proc. 10th Int. Workshop SPSC, oct. 2008, pp. $1-7$.

[12] S. K. Sharma, S. Chatzinotas, and B. Ottersten, "Exploiting polarization for spectrum sensing in cognitive satcoms," in Proc. 7th Int. Conf. CROWNCOM, June 2012.

[13] R. Nabar, H. Bolcskei, V. Erceg, D. Gesbert, and A. Paulraj, "Performance of multiantenna signaling techniques in the presence of polarization diversity," IEEE Trans. Signal Process., vol. 50, no. 10, pp. $2553-2562$, oct 2002 .

[14] C. Oestges, B. Clerckx, M. Guillaud, and M. Debbah, "Dual-polarized wireless communications: from propagation models to system performance evaluation," IEEE Trans. Wireless Commun., vol. 7, no. 10, pp. 4019 -4031, october 2008.

[15] FCC, "Mobile broadband: The benefits of additional spectrum," Tech. Rep. 6, Oct. 2010.

[16] W. Lee and Y. Yeh, "Polarization diversity system for mobile radio," IEEE Trans. Commun., vol. 20, no. 5, pp. 912 - 923, oct 1972.

[17] B. Lindmark and M. Nilsson, "On the available diversity gain from different dual-polarized antennas," IEEE J. Select. Areas Commun., vol. 19 , no. 2 , pp. $287-294$, feb 2001.

[18] S. K. Sharma, S. Chatzinotas, and B. Ottersten, "Satellite cognitive communications: Interference modeling and techniques selection," in 6th ASMS Conf., Sept. 2012, accepted.

[19] R. K. Sharma and J. W. Wallace, "Analysis of fusion and combining for wireless source detection,"' in Int. ITG workshop on Smart AntennasWSA, Feb. 16-18, Berlin 2009.

[20] H. Urkowitz, "Energy detection of unknown deterministic signals," Proc. of the IEEE, vol. 55, no. 4, pp. 523 - 531, April 1967.

[21] S. Atapattu, C. Tellambura, and H. Jiang, "Performance of an energy detector over channels with both multipath fading and shadowing," IEEE Trans. Wireless Commun., vol. 9, no. 12, pp. $3662-3670$, december 2010.

[22] D. Roddy, Satellite Communications, 4th ed. McGraw-Hill, 2006.

[23] S. M. Kay, Fundamentals of Statistical Signal Processing: Detection Theory, 1st ed., 1998.

[24] A. Nuttall, "Some integrals involving the function (corresp.)," IEEE Trans. Inf. Th., vol. 21, no. 1, pp. 95 - 96, Jan 1975. 